\title{
Characterization of Periodic Eigenfunctions of the Fourier Transform Operator
}

\author{
Comlan de Souza ${ }^{1}$, David W. Kammler ${ }^{2}$ \\ ${ }^{1}$ Department of Mathematics, California State University at Fresno, Fresno, USA \\ ${ }^{2}$ Department of Mathematics, Southern Illinois University at Carbondale, Carbondale, USA \\ Email: csouza@csufresno.edu,dkammler@siu.edu
}

Received October 4, 2013; revised November 4, 2013; accepted November 11, 2013

Copyright (C) 2013 Comlan de Souza, David W. Kammler. This is an open access article distributed under the Creative Commons Attribution License, which permits unrestricted use, distribution, and reproduction in any medium, provided the original work is properly cited.

\section{ABSTRACT}

Let the generalized function (tempered distribution) $f$ on $\mathbb{R}$ be a $p$-periodic eigenfunction of the Fourier transform operator $\mathcal{F}$, i.e., $f(x+p)=f(x), \mathcal{F} f=\lambda f$, for some $\lambda \in \mathbb{C}$. We show that $\lambda=1,-i,-1$, or $+i$, that $p=\sqrt{N}$ for some $N=1,2, \cdots$, and that $f$ has the representation $f(x)=\sum_{m=-\infty}^{\infty} \sum_{n=0}^{N-1} \gamma[n] \delta\left(x-\frac{n}{p}-m p\right)$ where $\delta$ is the Dirac functional and $\gamma$ is an eigenfunction of the discrete Fourier transform operator $\mathcal{F}_{N}$ with $\left(\mathcal{F}_{N} \gamma\right)[k]=\frac{1}{N} \sum_{n=0}^{N-1} \gamma[n] \mathrm{e}^{-2 \pi i k n / N}=\frac{\lambda}{\sqrt{N}} \gamma[k], \quad k=0,1, \cdots, N-1$. We generalize this result to $p_{1}, p_{2}$-periodic eigenfunctions of $\mathcal{F}$ on $\mathbb{R}^{2}$ and to $p_{1}, p_{2}, p_{3}$-periodic eigenfunctions of $\mathcal{F}$ on $\mathbb{R}^{3}$.

Keywords: Eigenfunction; Fourier Transform Operator

\section{Introduction}

In this paper, we will study certain generalizations of the Dirac comb (or III functional, see [1])

$$
\operatorname{III}(x):=\sum_{n=-\infty}^{\infty} \delta(x-n)
$$

where $\delta$ is the Dirac functional. We work within the context of the Schwartz theory of distributions [2] as developed in [1,3-7]. For purposes of manipulation we use "function" notation for $\delta$, III and related functionals. Various useful proprieties of $\delta$ and III are developed in [1,3-5].

The III functional is used in the study of sampling, periodization, etc., see $[1,4,5]$. We will illustrate this process using a notation that can be generalized to an $n$-dimensional setting. Let $a_{1} \in \mathbb{R}$ with $a_{1} \neq 0$, and let $A_{1}:=\frac{1}{a_{1}}$. We define the lattice

$$
\mathcal{L}_{a_{1}}:=\left\{n a_{1}: n \in \mathbb{Z}\right\}
$$

and the corresponding $a_{1}$-periodic Dirac comb

$$
\operatorname{grid}_{a_{1}}(x):=\sum_{a \in \mathcal{L}_{a_{1}}} \delta(x-a) .
$$

The Fourier transform of the $a_{1}$-periodic Dirac comb is

$$
\operatorname{grid}_{a_{1}}^{\wedge}(s)=\left|A_{1}\right| \operatorname{grid}_{A_{1}}(s) .
$$

Let $g$ be any univariate distribution with compact support. We can periodize $g$ by writing

$$
f(x):=\operatorname{grid}_{a_{1}}(x) * g(x),
$$

where * represents the convolution product, to obtain the weakly convergent Fourier series

$$
f(x)=\sum_{k=-\infty}^{\infty}\left|A_{1}\right| g^{\wedge}\left(k A_{1}\right) \mathrm{e}^{2 \pi i k A_{1} x} .
$$


We observe that $\operatorname{grid}_{a_{1}}$ has support at the points $n a_{1}, n=0, \pm 1, \pm 2, \cdots$ of the lattice $\mathcal{L}_{a_{1}}$, while the Fourier transform $\left|A_{1}\right| \operatorname{grid}_{A_{1}}$ has support at the points $\frac{n}{a_{1}}, n=0, \pm 1, \pm 2, \cdots$ of the lattice $\mathcal{L}_{A_{1}}$. It follows that

$$
\operatorname{grid}_{a_{1}}^{\wedge}=\operatorname{grid}_{a_{1}}
$$

if and only if

$$
a_{1}= \pm 1
$$

i.e., if and only if

$$
\operatorname{grid}_{a_{1}}=\text { III. }
$$

Let $\mathcal{F}$ be the Fourier transform operator on the space of tempered distributions. It is well known $[1,4,5]$, that $\mathcal{F}$ is linear and that

$$
\mathcal{F}^{4}=\mathcal{I},
$$

where $\mathcal{I}$ denotes the identity operator on the space of tempered distributions. We are interested in tempered distributions $f$ such that

$$
\mathcal{F} f=\lambda f,
$$

where $\lambda$ is a scalar. Any distribution $f$ that satisfies (8), and that we will call eigenfunction of $\mathcal{F}$, must also satisfy the following equation

$$
\mathcal{F}^{n} f=\lambda^{n} f, \quad n \in \mathbb{N}
$$

due to the linearity of the operator $\mathcal{F}$. When $n=4$, then $\mathcal{F}^{4} f=\lambda^{4} f$. Thus the eigenvalues of the operator $\mathcal{F}$ are $1,-1, i,-i$.

\section{Eigenvectors of $\mathcal{F}_{N}$}

We first consider the eigenvectors of the discrete Fourier transform operator $\mathcal{F}_{N}$ since, as we will see later, they can be used to construct all periodic eigenfunctions of the Fourier transform operator $\mathcal{F}[8,9]$.

Definition 1. Let $N=1,2, \cdots$. The matrix

$$
\mathcal{F}_{N}:=\frac{1}{N}\left[\begin{array}{ccccc}
1 & 1 & 1 & \cdots & 1 \\
1 & \omega & \omega^{2} & \cdots & \omega^{N-1} \\
1 & \omega^{2} & \omega^{4} & \cdots & \omega^{2 N-2} \\
\vdots & \vdots & \vdots & \ddots & \vdots \\
1 & \omega^{N-1} & \omega^{2 N-2} & \cdots & \omega^{(N-1)(N-1)}
\end{array}\right],
$$

$\omega:=\mathrm{e}^{-2 \pi i / N}$, is said to be the discrete Fourier transform operator.

It is easy to verify the operator identity

$$
\mathcal{F}_{N}^{2}=\frac{1}{N} \mathcal{R}_{N}
$$

where

$$
\mathcal{R}_{N}:=\left[\begin{array}{cccccc}
1 & 0 & 0 & \cdots & 0 & 0 \\
0 & 0 & 0 & \cdots & 0 & 1 \\
0 & 0 & 0 & \cdots & 1 & 0 \\
\vdots & \vdots & \vdots & \ddots & \vdots & \vdots \\
0 & 0 & 1 & \cdots & 0 & 0 \\
0 & 1 & 0 & \cdots & 0 & 0
\end{array}\right]
$$

is the reflection operator. It is easy to verify

$$
\mathcal{F}_{N}^{4}=\left[\frac{1}{N} \mathcal{R}_{N}\right]^{2}=\frac{1}{N^{2}} \mathcal{R}_{N}^{2}=\frac{1}{N^{2}} I_{N}
$$

where $I_{N}$ is the $N \times N$ identity matrix. In this way we see that if

$$
\mathcal{F}_{N} f=\lambda f, f \neq 0,
$$

then

$$
\lambda^{4}-\frac{1}{N^{2}}=0,
$$

so $\lambda$ must take one of the values $\pm 1 / \sqrt{N}, \pm i / \sqrt{N}$.

Let $M_{r}(N)$ be the multiplicity of the eigenvalue

$$
\lambda=\frac{(-i)^{r}}{\sqrt{N}}
$$

of $\mathcal{F}_{N}, r=0,1,2,3$, and let

$$
f_{N, r, \mu}[n], \mu=1,2, \cdots, M_{r}(N)
$$

be orthonormal eigenvectors of $\mathcal{F}_{N}$ corresponding to the eigenvalue

$$
\lambda=\frac{(-i)^{r}}{\sqrt{N}}, r=0,1,2,3 .
$$

Example 1. $N=2$

The matrix

$$
\mathcal{F}_{2}=\frac{1}{2}\left[\begin{array}{cc}
1 & 1 \\
1 & -1
\end{array}\right]
$$

has the eigenvalues $\lambda_{1}=1 / \sqrt{2}, \lambda_{2}=-1 / \sqrt{2}$ with corresponding eigenvectors

$$
\left[\begin{array}{c}
1 \\
-1+\sqrt{2}
\end{array}\right],\left[\begin{array}{c}
1 \\
-1-\sqrt{2}
\end{array}\right] .
$$

We normalize these vectors to obtain

$$
\begin{aligned}
& f_{2,0,1}[0]=\frac{1}{\sqrt{4-2 \sqrt{2}}}, f_{2,0,1}[1]=\frac{-1+\sqrt{2}}{\sqrt{4-2 \sqrt{2}}}, \\
& f_{2,2,1}[0]=\frac{1}{\sqrt{4+2 \sqrt{2}}}, f_{2,2,1}[1]=-\frac{1+\sqrt{2}}{\sqrt{4+2 \sqrt{2}}} .
\end{aligned}
$$




\section{The Main Results}

A generalized function $f, f \neq 0$, is said to be an eigenfunction of the Fourier transform operator $\mathcal{F}$ if

$$
\mathcal{F} f=\lambda f
$$

For $\lambda= \pm 1, \pm i$. We would like to characterize all periodic eigenfunctions $f$ of the Fourier transform operator $\mathcal{F}$, i.e.,

$$
\mathcal{F} f=\lambda f, f \neq 0,
$$

within the context of 1,2,3 dimensions.

\subsection{Periodic Eigenfunctions of $\mathcal{F}$ or $\mathbb{R}$}

Let $f$ be a $p$-periodic generalized function on $\mathbb{R}$, $p>0$, and assume that

$$
F:=\mathcal{F} f=\lambda f
$$

where $\lambda= \pm 1, \pm i$ and $f \neq 0$. The 2-periodic function

$$
\begin{aligned}
& f(x)= \\
& \frac{1}{2}\left\{\mathrm{III}\left(\frac{x}{2}\right)+\mathrm{III}\left(\frac{x-1 / 2}{2}\right)-\mathrm{III}\left(\frac{x-2 / 2}{2}\right)+\mathrm{III}\left(\frac{x-3 / 2}{2}\right)\right\},
\end{aligned}
$$

is such an eigenfunction, constructed from the eigenvector $f_{4,0,2}$ of $\mathcal{F}_{4}$. We will now characterize all such periodic eigenfunctions.

Since $f$ is $p$-periodic, $f$ is represented by its weakly convergent Fourier series

$$
f(x)=\sum_{k=-\infty}^{\infty} \Gamma[k] \mathrm{e}^{2 \pi i k x / p}
$$

We Fourier transform term by term to obtain the weakly convergent series

$$
F(s)=\sum_{k=-\infty}^{\infty} \Gamma[k] \delta\left(s-\frac{k}{p}\right)
$$

for the Fourier transform of $f$. Now since $F=\lambda f$ and $\lambda \neq 0, F$ must also be $p$ - periodic with

$$
F(s)=\left\{\sum_{0 \leq k<p^{2}} \Gamma[k] \delta\left(s-\frac{k}{p}\right)\right\} * \sum_{m=-\infty}^{\infty} \delta(s-m p)=\left\{\sum_{0 \leq k<p^{2}} \Gamma[k] \delta\left(s-\frac{k}{p}\right)\right\} * \frac{1}{p}\left(\frac{s}{p}\right) .
$$

We recognize this as the Fourier transform of

$$
\begin{aligned}
f(x) & =\left\{\sum_{0 \leq k<p^{2}} \Gamma[k] \mathrm{e}^{2 \pi i k x / p}\right\}(p x)=\left\{\sum_{0 \leq k<p^{2}} \Gamma[k] \mathrm{e}^{2 \pi i k x / p}\right\} \frac{1}{p} \sum_{n=-\infty}^{\infty} \delta\left(x-\frac{n}{p}\right) \\
& =\frac{1}{p} \sum_{n=-\infty}^{\infty} \sum_{0 \leq k<p^{2}} \Gamma[k] \mathrm{e}^{2 \pi i k x / p} \delta\left(x-\frac{n}{p}\right)=\frac{1}{p} \sum_{n=-\infty}^{\infty}\left\{\sum_{0 \leq k<p^{2}} \Gamma[k] \mathrm{e}^{2 \pi i k n / p^{2}}\right\} \delta\left(x-\frac{n}{p}\right) .
\end{aligned}
$$

We define

$$
\gamma[n]:=\sum_{0 \leq k<p^{2}} \Gamma[k] \mathrm{e}^{2 \pi i k n / p^{2}}
$$

$$
\frac{n^{\prime}}{p}=\frac{p^{2}+n}{p},
$$

and write

$$
f(x)=\frac{1}{p} \sum_{n=-\infty}^{\infty} \gamma[n] \delta\left(x-\frac{n}{p}\right) .
$$

Now if the term

$$
\gamma[n] \delta\left(x-\frac{n}{p}\right), \gamma[n] \neq 0
$$

appears in the sum (13) then (since $f$ is $p$-periodic)

$$
\gamma(n) \delta\left(x-p-\frac{n}{p}\right)
$$

must also appear. Thus

$$
\gamma\left[n^{\prime}\right] \delta\left(x-\frac{n^{\prime}}{p}\right)=\gamma[n] \delta\left(x-\frac{p^{2}+n}{p}\right)
$$

for some integer $n^{\prime}$. It follows that

i.e,

$$
p^{2}=n^{\prime}-n,
$$

and

$$
\gamma[n]=\gamma\left[n^{\prime}\right] .
$$

thus

$$
p^{2}=N
$$

for some $N=1,2, \cdots$, and since $\gamma[n]$ is $N$-periodic, we can use (13) to write

$$
\begin{aligned}
f(x) & =\frac{1}{\sqrt{N}} \sum_{n=-\infty}^{\infty} \gamma[n] \delta\left(x-\frac{n}{\sqrt{N}}\right) \\
& =\frac{1}{\sqrt{N}} \sum_{m=-\infty}^{\infty} \sum_{n=0}^{N-1} \gamma[n] \delta\left(x-\frac{n}{\sqrt{N}}-m \sqrt{N}\right)
\end{aligned}
$$

where 


$$
\gamma[n]=\sum_{k=0}^{N-1} \Gamma[k] \mathrm{e}^{2 \pi i k n / N}
$$

is the inverse Fourier transform of the $N$-periodic sequence of Fourier coefficients $\Gamma$. Since $F=\lambda f$ we can use (12), (14) to see that

$$
\Gamma[k]=\left(\mathcal{F}_{N} \gamma\right)[k]=\frac{\lambda}{\sqrt{N}} \gamma[k], k=0,1, \cdots, N-1,
$$

i.e., that $\gamma$ is an eigenvector of the discrete Fourier transform operator $\mathcal{F}_{N}$ associated with the eigenvalue $\frac{\lambda}{\sqrt{N}}$. In this way we prove the following

Theorem 1. Let the generalized function $f$ on $\mathbb{R}$ be a $p$-periodic eigenfunction of the Fourier transform operator $\mathcal{F}$ with eigenvalue $\lambda=1,-i,-1$, or $+i$. Then $p=\sqrt{N}$ for some integer $N=1,2, \cdots$ and $f$ has the representation

$$
f(x)=\sum_{m=-\infty}^{\infty} \sum_{n=0}^{N-1} \gamma[n] \delta\left(x-\frac{n}{p}-m p\right)
$$

where $\gamma$ is an eigenvector of the discrete Fourier transform operator $\mathcal{F}_{N}$ with

$$
\begin{aligned}
& \left(\mathcal{F}_{N} \gamma\right)[k] \\
& =\frac{1}{N} \sum_{n=0}^{N-1} \gamma[n] \mathrm{e}^{-2 \pi i k n / N} \\
& =\frac{\lambda}{\sqrt{N}} \gamma[k], k=0,1, \cdots, N-1 .
\end{aligned}
$$

Example 2. When $N=1$ we obtain the corresponding 1-periodic

$$
f(x)=\sum_{n=-\infty}^{\infty} \delta(x-n)=\operatorname{III}(x),
$$

with

$$
\mathcal{F} \text { III }=\text { III. }
$$

Of course, this particular result is well known, see [1]. Our argument shows that a periodic eigenfunction of the Fourier transform operator that has one singular point per unit cell must be a scalar multiple of the Dirac comb III .

Example 3. When $N=2$, we obtain the $\sqrt{2}$-periodic eigenfunctions

$$
\begin{aligned}
f_{1}(x) & =\frac{1}{\sqrt{4-2 \sqrt{2}}} \sum_{n=-\infty}^{\infty} \delta(x-n \sqrt{2})+\frac{-1+\sqrt{2}}{\sqrt{4-2 \sqrt{2}}} \sum_{n=-\infty}^{\infty} \delta\left(x-\frac{1}{\sqrt{2}}-n \sqrt{2}\right) \\
& =\frac{1}{\sqrt{2(4-2 \sqrt{2})}}\left(\frac{x}{\sqrt{2}}\right)+\frac{-1+\sqrt{2}}{\sqrt{2(4-2 \sqrt{2})}}\left(\frac{x}{\sqrt{2}}-\frac{1}{2}\right),
\end{aligned}
$$

and

$$
\begin{aligned}
f_{2}(x) & =\frac{1}{\sqrt{4+2 \sqrt{2}}} \sum_{n=-\infty}^{\infty} \delta(x-n \sqrt{2})-\frac{1+\sqrt{2}}{\sqrt{4+2 \sqrt{2}}} \sum_{n=-\infty}^{\infty} \delta\left(x-\frac{1}{\sqrt{2}}-n \sqrt{2}\right) \\
& =\frac{1}{\sqrt{2(4+2 \sqrt{2})}}\left(\frac{x}{\sqrt{2}}\right)-\frac{1+\sqrt{2}}{\sqrt{2(4+2 \sqrt{2})}}\left(\frac{x}{\sqrt{2}}-\frac{1}{2}\right)
\end{aligned}
$$

from the eigenvectors $f_{2,0,1}$ and $f_{2,2,1}$ for $\mathcal{F}_{2}$. It is easy to verify that

$$
\left(\mathcal{F} f_{1}\right)(s)=f_{1}(s),\left(\mathcal{F} f_{2}\right)(s)=-f_{2}(s) .
$$

\section{Characterization of periodic eigenfunctions of $\mathcal{F}$} on $\mathbb{R}^{2}$

Let $f$ be a bivariate generalized function and assume that $f$ is an eigenfunction of $\mathcal{F}$, i.e.,

$$
F:=\mathcal{F f}=\lambda f
$$

with $\lambda=1,-i,-1$, or $+i$, (and $f \neq 0)$. Assume further that $f$ is $a_{1}, a_{2}$-periodic, i.e.,

$$
f\left(x+a_{1}\right)=f(x), f\left(x+a_{2}\right)=f(x) .
$$

Here $a_{1}, a_{2}$ are linearly independent vectors in $\mathbb{R}^{2}$.
We simplify the analysis by rotating the coordinate system as necessary so as to place a shortest vector from the lattice $\mathcal{L}_{a_{1}, a_{2}}$ along the positive $x$-axis. We can and do further assume with no loss of generality that $a_{1}, a_{2}$ have the form

$$
a_{1}=\left(\alpha_{1}, 0\right)^{\mathrm{T}}, a_{2}=\left(\beta_{1}, \beta_{2}\right)^{\mathrm{T}}
$$

where

$$
\begin{gathered}
\alpha_{1}>0 \\
\alpha_{1}^{2} \leq \beta_{1}^{2}+\beta_{2}^{2} \\
\beta_{2}>0 \\
0 \leq \beta_{1}<\alpha_{1} .
\end{gathered}
$$


The dual vectors then have the representation

$$
A_{1}=\frac{1}{\alpha_{1} \beta_{2}}\left(\beta_{2},-\beta_{1}\right)^{\mathrm{T}}, A_{2}=\frac{1}{\alpha_{1} \beta_{2}}\left(0, \alpha_{1}\right)^{\mathrm{T}},
$$

and

$$
\operatorname{grid}_{a_{1}, a_{2}}(x)=\sum_{n_{1}=-\infty n_{2}=-\infty}^{\infty} \sum^{\infty} \delta\left(x-n_{1} a_{1}-n_{2} a_{2}\right)
$$

has the Fourier transform

$$
\operatorname{grid}_{a_{1}, a_{2}}^{\wedge}(s)=\Delta \sum_{k_{1}=-\infty}^{\infty} \sum_{k_{2}=-\infty}^{\infty} \delta\left(s-k_{1} A_{1}-k_{2} A_{2}\right)
$$

where $\Delta=\left|\operatorname{det}\left(A_{1}, A_{2}\right)\right|$. Now since $f$ is $a_{1}, a_{2}$-periodic, $f$ can be represented by the weakly convergent Fourier series

$$
f(x)=\sum_{k_{1}=-\infty}^{\infty} \sum_{k_{2}=-\infty}^{\infty} \Gamma\left[k_{1}, k_{2}\right] \mathrm{e}^{2 \pi i x \cdot\left(k_{1} A_{1}+k_{2} A_{2}\right)} .
$$

We Fourier transform the series (20) to obtain the weakly convergent series

$$
F(s)=\sum_{k_{1}=-\infty}^{\infty} \sum_{k_{2}=-\infty}^{\infty} \Gamma\left[k_{1}, k_{2}\right] \delta\left(s-k_{1} A_{1}-k_{2} A_{2}\right) .
$$

From (21), we see that the support of $F$ lies on the lattice $\mathcal{L}_{A_{1}, A_{2}}$ and since $F=\lambda f, F$ must also be $a_{1}, a_{2}$-periodic so we can write

$$
\begin{aligned}
& F(s) \\
& =\left\{\sum_{k_{1} A_{1}+k_{2} A_{2} \in \mathcal{U}} \Gamma\left[k_{1}, k_{2}\right] \delta\left(s-k_{1} A_{1}-k_{2} A_{2}\right)\right\} * \operatorname{grid}_{a_{1}, a_{2}}(s)
\end{aligned}
$$

where

$$
\mathcal{U}:=\left\{x_{1}^{\prime} a_{1}+x_{2}^{\prime} a_{2}: 0 \leq x_{1}^{\prime}<1,0 \leq x_{2}^{\prime}<1\right\}
$$

is a primitive unit cell associated with the lattice $\mathcal{L}_{a_{1}, a_{2}}$, where $x_{1}^{\prime}, x_{2}^{\prime}$ are affine coordinates, and $*$ is the bivariate convolution product. Using the bivariate inverse Fourier transform, we see that

$$
\begin{aligned}
& f(x)=F(x) \cdot \operatorname{grid}_{a_{1}, a_{2}}^{\wedge}(x) \\
= & \Delta \sum_{n_{1}=-\infty}^{\infty} \sum_{n_{2}=-\infty}^{\infty} \sum_{k_{1} A_{1}+k_{2} A_{2} \in \mathcal{U}}\left\{\Gamma\left[k_{1}, k_{2}\right] \cdot \mathrm{e}^{2 \pi i\left(k_{1} A_{1}+k_{2} A_{2}\right) \cdot\left(n_{1} A_{1}+n_{2} A_{2}\right)} \cdot \delta\left(x-n_{1} A_{1}-n_{2} A_{2}\right)\right\} \\
= & \frac{1}{\alpha_{1} \beta_{2}} \sum_{n_{1}=-\infty}^{\infty} \sum_{n_{2}=-\infty}^{\infty}\left\{\left\{\sum_{k_{1} A_{1}+k_{2} A_{2} \in \mathcal{U}} \Gamma\left[k_{1}, k_{2}\right] \cdot \mathrm{e}^{2 \pi i\left(\left(\beta_{1}^{2}+\beta_{2}^{2}\right) n_{1} k_{1}-\alpha_{1} \beta_{1}\left(n_{1} k_{2}+n_{2} k_{1}\right)+\alpha_{1}^{2} n_{2} k_{2}\right\} /\left(\alpha_{1}^{2} \beta_{2}^{2}\right)}\right\}\right. \\
& \left.\cdot \delta\left(x_{1}-\frac{n_{1} \beta_{2}}{\alpha_{1} \beta_{2}}, x_{2}+\frac{n_{1} \beta_{1}-n_{2} \alpha_{1}}{\alpha_{1} \beta_{2}}\right)\right\} .
\end{aligned}
$$

We define

$$
\begin{aligned}
& \gamma\left[n_{1}, n_{2}\right]:=\sum_{k_{1} A_{1}+k_{2} A_{2} \in \mathcal{U}}\left\{\Gamma\left[k_{1}, k_{2}\right]\right. \\
& \left.\cdot \mathrm{e}^{2 \pi i\left(\left(\beta_{1}^{2}+\beta_{2}^{2}\right) n_{1} k_{1}-\alpha_{1} \beta_{1}\left(n_{1} k_{2}+n_{2} k_{1}\right)+\alpha_{1}^{2} n_{2} k_{2}\right\} /\left(\alpha_{1}^{2} \beta_{2}^{2}\right)}\right\}
\end{aligned}
$$

and write

$$
\begin{aligned}
f\left(x_{1}, x_{2}\right)= & \frac{1}{\alpha_{1} \beta_{2}} \sum_{n_{1}=-\infty}^{\infty} \sum_{n_{2}=-\infty}^{\infty}\left\{\gamma\left[n_{1}, n_{2}\right]\right. \\
& \left.\cdot \delta\left(x_{1}-\frac{n_{1} \beta_{2}}{\alpha_{1} \beta_{2}}, x_{2}+\frac{n_{1} \beta_{1}-n_{2} \alpha_{1}}{\alpha_{1} \beta_{2}}\right)\right\}
\end{aligned}
$$

Now $f$ is $a_{1}, a_{2}$-periodic, so if $\gamma\left[n_{1}, n_{2}\right] \neq 0$ for some integers $n_{1}, n_{2}$, then the term

$$
\gamma\left[n_{1}, n_{2}\right] \delta\left(x_{1}-\alpha_{1}-\frac{n_{1} \beta_{2}}{\alpha_{1} \beta_{2}}, x_{2}+\frac{n_{1} \beta_{1}-n_{2} \alpha_{1}}{\alpha_{1} \beta_{2}}\right)
$$

equals the term

$$
\gamma\left[n_{1}^{\prime}, n_{2}^{\prime}\right] \delta\left(x_{1}-\frac{n_{1}^{\prime} \beta_{2}}{\alpha_{1} \beta_{2}}, x_{2}+\frac{n_{1}^{\prime} \beta_{1}-n_{2}^{\prime} \alpha_{1}}{\alpha_{1} \beta_{2}}\right)
$$

and the term

$$
\gamma\left[n_{1}, n_{2}\right] \delta\left(x_{1}-\beta_{1}-\frac{n_{1} \beta_{2}}{\alpha_{1} \beta_{2}}, x_{2}-\beta_{2}+\frac{n_{1} \beta_{1}-n_{2} \alpha_{1}}{\alpha_{1} \beta_{2}}\right)
$$

equals the term

$$
\gamma\left[n_{1}^{\prime \prime}, n_{2}^{\prime \prime}\right] \delta\left(x_{1}-\frac{n_{1}^{\prime \prime} \beta_{2}}{\alpha_{1} \beta_{2}}, x_{2}+\frac{n_{1}^{\prime \prime} \beta_{1}-n_{2}^{\prime \prime} \alpha_{1}}{\alpha_{1} \beta_{2}}\right)
$$

for some integers $n_{1}^{\prime}, n_{2}^{\prime}, n_{1}^{\prime \prime}, n_{2}^{\prime \prime}$. From the supports of these $\delta$-functions we see that

$$
\alpha_{1}+\frac{n_{1} \beta_{2}}{\alpha_{1} \beta_{2}}=\frac{n_{1}^{\prime} \beta_{2}}{\alpha_{1} \beta_{2}},
$$

i.e.,

$$
\begin{aligned}
& \alpha_{1}^{2}=n_{1}^{\prime}-n_{1} \\
& \alpha_{1}^{2}=N_{1}
\end{aligned}
$$

for some $N_{1}=1,2, \cdots \quad$. Likewise, we see in turn that 


$$
\begin{aligned}
& n_{1} \beta_{1}-n_{2} \alpha_{1}=n_{1}^{\prime} \beta_{1}-n_{2}^{\prime} \alpha_{1}, \\
& \left(n_{1}^{\prime}-n_{1}\right) \beta_{1}=\left(n_{2}^{\prime}-n_{2}\right) \alpha_{1}, \\
& \alpha_{1}^{2} \beta_{1}=\left(n_{2}^{\prime}-n_{2}\right) \alpha_{1}, \\
& \alpha_{1} \beta_{1}=n_{2}^{\prime}-n_{2}=M
\end{aligned}
$$

for some $M=0, \pm 1, \pm 2, \cdots$, and analogously

$$
\begin{aligned}
& \beta_{1}+\frac{n_{1} \beta_{2}}{\alpha_{1} \beta_{2}}=\frac{n_{1}^{\prime \prime} \beta_{2}}{\alpha_{1} \beta_{2}}, \\
& \alpha_{1} \beta_{1}=n_{1}^{\prime \prime}-n_{1}=M .
\end{aligned}
$$

Finally,

$$
\begin{aligned}
& \beta_{2}-\frac{n_{1} \beta_{1}-n_{2} \alpha_{1}}{\alpha_{1} \beta_{2}}=-\frac{n_{1}^{\prime \prime} \beta_{1}-n_{2}^{\prime \prime} \alpha_{1}}{\alpha_{1} \beta_{2}}, \\
& \beta_{2}^{2}+\left(n_{1}^{\prime \prime}-n_{1}\right) \frac{\beta_{1}}{\alpha_{1}}=n_{2}^{\prime \prime}-n_{2}, \\
& \beta_{2}^{2}+\alpha_{1} \beta_{1} \frac{\beta_{1}}{\alpha_{1}}=n_{2}^{\prime \prime}-n_{2}, \\
& \beta_{2}^{2}+\beta_{1}^{2}=N_{2}
\end{aligned}
$$

for some $N_{2}=1,2, \cdots$. Using these expressions we can now write

$$
\begin{aligned}
& \alpha_{1}=\sqrt{N_{1}}, \beta_{1}=\frac{M}{\sqrt{N_{1}}} \\
& \beta_{2}=\frac{\sqrt{N_{1} N_{2}-M^{2}}}{\sqrt{N_{1}}} \\
& a_{1}=\frac{1}{\sqrt{N_{1}}}\left(N_{1}, 0\right)^{\mathrm{T}} \\
& a_{2}=\frac{1}{\sqrt{N_{1}}}\left(M, \sqrt{N_{1} N_{2}-M^{2}}\right)^{\mathrm{T}} \\
& A_{1}=\frac{1}{\sqrt{N_{1}\left(N_{1} N_{2}-M^{2}\right)}}\left(\sqrt{N_{1} N_{2}-M^{2}},-M\right)^{\mathrm{T}} \\
& A_{2}=\frac{1}{\sqrt{N_{1}\left(N_{1} N_{2}-M^{2}\right)}}\left(0, N_{1}\right)^{\mathrm{T}}
\end{aligned}
$$

where, in view of (16)-(19)

$$
N_{1} \leq N_{2}, \quad 0 \leq M<N_{1}
$$

and

$$
\left\|a_{1}\right\|=\sqrt{N_{1}}, \quad\left\|a_{2}\right\|=\sqrt{N_{2}} .
$$

From (21), (23) we also have

$$
\begin{gathered}
\gamma\left[n_{1}, n_{2}\right]=\sum_{k_{1} A_{1}+k_{2} A_{2} \in \mathcal{U}}\left\{\Gamma\left[k_{1}, k_{2}\right]\right. \\
\left.\cdot \mathrm{e}^{2 \pi i\left\{N_{2} n_{1} k_{1}-M\left(n_{1} k_{2}+n_{2} k_{1}\right)+N_{1} n_{2} k_{2} /\left(N_{2} N_{1}-M^{2}\right)\right\}}\right\}
\end{gathered}
$$

$$
\begin{array}{r}
F(s)=\sum_{k_{1}=-\infty}^{\infty} \sum_{k_{2}=-\infty}^{\infty} \Gamma\left[k_{1}, k_{2}\right] \delta\left(s-k_{1} A_{1}-k_{2} A_{2}\right) \\
=\sum_{k_{1}=-\infty}^{\infty} \sum_{k_{2}=-\infty}^{\infty}\left\{\Gamma [ k _ { 1 } , k _ { 2 } ] \cdot \delta \left(s_{1}-\frac{k_{1}}{\sqrt{N_{1}}},\right.\right. \\
\left.\left.s_{2}+\frac{k_{1} M-k_{2} N_{1}}{\sqrt{N_{1}\left(N_{1} N_{2}-M^{2}\right)}}\right)\right\} .
\end{array}
$$

We will now consider separately the cases $M=0, M>0$.

Case $M=0$

When $M=0$ the vectors $a_{1}, a_{2}$ are orthogonal and $f$ has the corresponding periods

$$
\alpha_{1}=\sqrt{N_{1}}, \beta_{2}=\sqrt{N_{2}},
$$

along the $x$-axis and $y$-axis, respectively. The function $\gamma$ is represented by the synthesis equation

$$
\gamma\left[n_{1}, n_{2}\right]=\sum_{k_{1}=0}^{N_{1}-1 k_{2}=0}\left\{\Gamma\left[k_{1}, k_{2}\right] \cdot e^{2 \pi i\left(n_{1} k_{1} / N_{1}+n_{2} k_{2} / N_{2}\right)}\right\},
$$

and by using (24) and (26), in turn we write

$$
\begin{gathered}
\quad F\left(s_{1}, s_{2}\right) \\
=\sum_{k_{1}=-\infty}^{\infty} \sum_{k_{2}=-\infty}^{\infty}\left\{\Gamma\left[k_{1}, k_{2}\right] \cdot \delta\left(s_{1}-\frac{k_{1}}{\sqrt{N_{1}}}, s_{2}-\frac{k_{2}}{\sqrt{N_{2}}}\right)\right\} \\
=\lambda f\left(s_{1}, s_{2}\right)=\frac{\lambda}{\sqrt{N_{1} N_{2}}} \sum_{n_{1}=-\infty n_{2}=-\infty}^{\infty}\left\{\gamma\left[n_{1}, n_{2}\right]\right. \\
\left.\cdot \delta\left(s_{1}-\frac{k_{1}}{\sqrt{N_{1}}}, s_{2}-\frac{k_{2}}{\sqrt{N_{2}}}\right)\right\} .
\end{gathered}
$$

In this way we conclude that

$$
\Gamma\left[k_{1}, k_{2}\right]=\frac{\lambda}{\sqrt{N_{1} N_{2}}} \gamma\left[k_{1}, k_{2}\right] .
$$

Thus $\gamma$ must be an eigenvector of the bivariate discrete Fourier transform $\mathcal{F}_{N_{1}, N_{2}}$ associated with the eigenvalue $\frac{\lambda}{\sqrt{N_{1} N_{2}}},(\lambda=1,-i,-1$, or $+i)$. Since $\gamma$ is an $N_{1}, N_{2}$-periodic sequence of complex numbers, we can write

$$
\begin{aligned}
f(x)= & \sum_{m_{1}=-\infty m_{2}=-\infty}^{\infty} \sum_{n_{1}=0}^{\infty} \sum_{n_{2}=0}^{N_{1}-1 N_{2}-1}\left\{\gamma\left[n_{1}, n_{2}\right]\right. \\
& \left.\cdot \delta\left(x_{1}-\frac{n_{1}}{\sqrt{N_{1}}}-m_{1} \sqrt{N_{1}}, x_{2}-\frac{n_{2}}{\sqrt{N_{2}}}-m_{2} \sqrt{N_{2}}\right)\right\} .
\end{aligned}
$$


Case $M \neq 0$

We observe that

$$
\begin{aligned}
& a_{1}=\frac{1}{\sqrt{N_{1}}}\left(N_{1}, 0\right)^{\mathrm{T}}, \\
& \begin{aligned}
N_{1} a_{2}-M a_{1} & =\sqrt{N_{1}}\left(M, \sqrt{N_{1} N_{2}-M^{2}}\right)^{\mathrm{T}}-M\left(\sqrt{N_{1}}, 0\right)^{\mathrm{T}} \\
& =\frac{1}{\sqrt{N_{1}}}\left(0, N_{1} \sqrt{N_{1} N_{2}-M^{2}}\right)^{\mathrm{T}} .
\end{aligned}
\end{aligned}
$$

Since $f$ is $a_{1}, a_{2}$-periodic, then $f$ is also $a_{1}, N_{1} a_{2}-M a_{1}$-periodic. Thus $f$ has the periods

$$
\alpha_{1}=\sqrt{N_{1}} \text {, and } \beta_{2}^{\prime}=\sqrt{N_{1}\left(N_{1} N_{2}-M^{2}\right)}
$$

along the $x$-axis and the $y$-axis, respectively, a situation covered by the analysis from the $M=0$ case. In this way we prove

Theorem 2. Let the generalized function $f$ on $\mathbb{R}^{2}$ be an $a_{1}, a_{2}$-periodic eigenfunction of the Fourier transform operator $\mathcal{F}$ with eigenvalue $\lambda=1,-i,-1$, or $+i$. Assume that the linearly independent periods $a_{1}, a_{2}$ from $\mathbb{R}^{2}$ have been chosen as small as possible subject to the constraint that $0<\left\|a_{1}\right\| \leq\left\|a_{2}\right\|$. Then there are positive integers $N_{1} \leq N_{2}$ such that

$$
\left\|a_{1}\right\|=\sqrt{N_{1}},\left\|a_{2}\right\|=\sqrt{N_{2}}
$$

and there is a nonnegative integer $M<N_{1}$ such that $a_{1}$ is orthogonal to

$$
a_{2}^{\prime}:=N_{1} a_{2}-M a_{1}
$$

with

$$
\left\|a_{2}^{\prime}\right\|=\sqrt{N_{2}^{\prime}}, N_{2}^{\prime}:=N_{1}\left(N_{1} N_{2}-M^{2}\right) .
$$

The generalized function $f$ is $a_{1}, a_{2}^{\prime}$-periodic and there is an orthogonal transformation $Q$ such that

$$
f_{Q}(x):=f(Q x)
$$

is $\left(\sqrt{N_{1}}, 0\right)^{\mathrm{T}},\left(0, \sqrt{N_{2}^{\prime}}\right)^{\mathrm{T}}$-periodic with the representation

$$
\begin{aligned}
& f_{Q}(x)=\sum_{m_{1}=-\infty}^{\infty} \sum_{m_{2}=-\infty}^{\infty} \sum_{n_{1}=0}^{N_{1}-1} \sum_{n_{2}=0}^{N_{2}^{\prime}-1} \gamma\left[n_{1}, n_{2}\right] \\
& \cdot \delta\left(x_{1}-\frac{n_{1}}{\sqrt{N_{1}}}-m_{1} \sqrt{N_{1}}, x_{2}-\frac{n_{2}}{\sqrt{N_{2}^{\prime}}}-m_{2} \sqrt{N_{2}^{\prime}}\right) .
\end{aligned}
$$

Here $\gamma$ is an eigenfunction of $\mathcal{F}_{N_{1}, N_{2}^{\prime}}$ with

$$
\begin{aligned}
& \left(\mathcal{F}_{N_{1}, N_{2}^{\prime}} \gamma\right)\left[k_{1}, k_{2}\right] \\
& =\frac{1}{N_{1} N_{2}^{\prime}} \sum_{n_{1}=0}^{N_{1}-1 N_{2}^{\prime}-1} \sum_{n_{2}=0}^{\gamma} \gamma\left[n_{1}, n_{2}\right] \cdot \mathrm{e}^{-2 \pi i\left(k_{1} n_{1} / N_{1}+k_{2} n_{2} / N_{2}^{\prime}\right)} \\
& =\frac{\lambda}{\sqrt{N_{1} N_{2}^{\prime}}} \gamma\left[k_{1}, k_{2}\right]
\end{aligned}
$$

for $0 \leq k_{1} \leq N_{1}-1,0 \leq k_{2} \leq N_{2}^{\prime}-1$.

Note that the $N_{1} N_{2}$ normalized eigenfunctions $\gamma$ denoted by

$$
f_{N_{1}, r_{1}, \mu_{1} ; N_{2}, r_{2}, \mu_{2}}\left[n_{1}, n_{2}\right]:=f_{N_{1}, r_{1}, \mu_{1}}\left[n_{1}\right] \cdot f_{N_{2}, r_{2}, \mu_{2}}\left[n_{2}\right],
$$

with $\mu_{k}=1, \cdots, M_{r_{k}}\left(N_{k}\right), k=1,2$ of $\mathcal{F}_{N_{1}, N_{2}}$ serve as an orthonormal basis for the $N_{1} N_{2}$ dimensional space $\mathbb{P}_{N_{1}, N_{2}}$ of $N_{1}, N_{2}$-periodic discrete real valued functions. Here (29) has the corresponding eigenvalue

$$
\lambda=\frac{(-i)^{r_{1}}}{\sqrt{N_{1}}} \frac{(-i)^{r_{2}}}{\sqrt{N_{2}}}, r_{1}, r_{2}=0,1,2,3 .
$$

Theorem 3. Let the generalized function $f$ on $\mathbb{R}^{3}$ be an $a_{1}, a_{2}, a_{3}$-periodic eigenfunction of the Fourier transform operator $\mathcal{F}$ with eigenvalue $\lambda=1,-i,-1$, or $+i$. Assume that the linearly independent periods $a_{1}, a_{2}, a_{3}$ from $\mathbb{R}^{3}$ have been chosen as small as possible subject to the constraint that

$0<\left\|a_{1}\right\| \leq\left\|a_{2}\right\| \leq\left\|a_{3}\right\|$. Then there are positive integers $N_{1} \leq N_{2} \leq N_{3}$ such that

$$
\left\|a_{1}\right\|=\sqrt{N_{1}},\left\|a_{2}\right\|=\sqrt{N_{2}},\left\|a_{3}\right\|=\sqrt{N_{3}}
$$

and there are nonnegative integers

$$
0 \leq M_{1}<N_{1}, 0 \leq M_{2}<N_{1}, 0 \leq M_{3}<N_{1}+N_{2}
$$

such that $a_{1}$,

$$
a_{2}^{\prime}:=N_{1} a_{2}-M_{1} a_{1}
$$

and

$$
\begin{aligned}
a_{3}^{\prime}:=N_{1}[ & \left(M_{1} M_{3}-N_{2} M_{2}\right) a_{1}-\left(N_{1} M_{3}-M_{1} M_{2}\right) a_{2} \\
& \left.+\left(N_{1} N_{2}-M_{1}^{2}\right) a_{3}\right]
\end{aligned}
$$

are pairwisely orthogonal with

$$
\left\|a_{2}^{\prime}\right\|=\sqrt{N_{2}^{\prime}},\left\|a_{3}^{\prime}\right\|=\sqrt{N_{3}^{\prime}}
$$

where

$$
\begin{gathered}
N_{2}^{\prime}:=N_{1}\left(N_{1} N_{2}-M_{1}^{2}\right), \\
N_{3}^{\prime}:=N_{1}^{2}\left(N_{1} N_{2}-M_{1}^{2}\right)\left[N_{1} N_{2} N_{3}+2 M_{1} M_{2} M_{3}\right. \\
\left.-\left(N_{1} M_{3}^{2}+N_{2} M_{2}^{2}+N_{3} M_{1}^{2}\right)\right]
\end{gathered}
$$

The generalized function $f$ is $a_{1}, a_{2}^{\prime}, a_{3}^{\prime}$-periodic, and there is an orthogonal transformation $Q$ such that

$$
f_{Q}(x):=f(Q x)
$$

is

$$
\left(\sqrt{N_{1}}, 0,0\right)^{\mathrm{T}},\left(0, \sqrt{N_{2}^{\prime}}, 0\right)^{\mathrm{T}},\left(0,0, \sqrt{N_{3}^{\prime}}\right)^{\mathrm{T}}
$$

-periodic with the representation 


$$
f_{Q}(x)=\sum_{m_{1}, m_{2}, m_{3}=-\infty}^{\infty} \sum_{n_{1}=0}^{N_{1}-1} \sum_{n_{2}=0}^{N_{2}^{\prime}-1} \sum_{n_{3}=0}^{N_{3}^{\prime}-1}\left\{\gamma\left[n_{1}, n_{2}, n_{3}\right] \cdot \delta\left(x_{1}-\frac{n_{1}}{\sqrt{N_{1}}}-m_{1} \sqrt{N_{1}}, x_{2}-\frac{n_{2}}{\sqrt{N_{2}^{\prime}}}-m_{2} \sqrt{N_{2}^{\prime}}, x_{3}-\frac{n_{3}}{\sqrt{N_{3}^{\prime}}}-m_{3} \sqrt{N_{3}^{\prime}}\right)\right\} .
$$

Here

$$
\left(\mathcal{F}_{N_{1}, N_{2}^{\prime}, N_{3}^{\prime}} \gamma\right)\left[k_{1}, k_{2}, k_{3}\right]=\sum_{n_{1}=0}^{N_{1}-1 n_{2}=0 n_{3}^{\prime}=0}\left\{\sigma \cdot \gamma\left[n_{1}, n_{2}, n_{3}\right] \cdot \mathrm{e}^{-2 \pi i\left(k_{1} n_{1} / N_{1}+k_{2} n_{2} / N_{2}^{\prime}+k_{3} n_{3} / N_{3}^{\prime}\right)}\right\}=\frac{\lambda}{\sqrt{N_{1} N_{2}^{\prime} N_{3}^{\prime}}} \gamma\left[k_{1}, k_{2}, k_{3}\right]
$$

where

$$
\sigma=\frac{1}{N_{1} N_{2}^{\prime} N_{3}^{\prime}}
$$

for

$$
0 \leq k_{1} \leq N_{1}-1,0 \leq k_{2} \leq N_{2}^{\prime}-1
$$

and

$$
0 \leq k_{3} \leq N_{3}^{\prime}-1 \text {. }
$$

\subsection{Some Quasiperiodic Eigenfunctions of the Fourier Transform Operator on $\mathbb{R}^{2}$}

In this section we will construct some quasiperiodic eigenfunctions of the Fourier transform operator. A generalized function $f$ is said to be quasiperiodic if the Fourier transform $f^{\wedge}$ is a weighted sum of Dirac $\delta$ functionals with isolated support [10].

Lemma 1 Let $a_{1}, a_{2}$ be linearly independent vectors in $\mathbb{R}^{2}$.If

$$
\left|\operatorname{det}\left[\begin{array}{ll}
a_{1} & a_{2}
\end{array}\right]\right|=1,
$$

and $\operatorname{grid}_{a_{1}, a_{2}}$ is distinct from $\operatorname{grid}_{a_{1}, a_{2}}^{\wedge}$, then

$$
\begin{aligned}
& f_{+}(x):=\operatorname{grid}_{a_{1}, a_{2}}(x)+\operatorname{grid}_{a_{1}, a_{2}}^{\wedge}(x) \\
& f_{-}(x):=\operatorname{grid}_{a_{1}, a_{2}}(x)-\operatorname{grid}_{a_{1}, a_{2}}^{\wedge}(x)
\end{aligned}
$$

are eigenfunctions of the Fourier transform operator $\mathcal{F}$ associated with $\lambda=1, \lambda=-1$, respectively.

Quasiperiodic eigenfunctions of $\mathcal{F}$ on $\mathbb{R}^{2}$ with $m$-fold rotational symmetry.

Let

$$
\alpha=1 / \sqrt{\sin \left(\frac{2 \pi}{n}\right)}
$$

for some $n=3,4, \cdots$, and let

$$
a_{k}=\alpha(\cos (2 \pi k / n), \sin (2 \pi k / n))^{\mathrm{T}}
$$

where $0 \leq k \leq n-1$, be the vertices of a regular $n$-gon with center at the origin. The parameter $\alpha$ has been chosen so that

$$
\operatorname{det}\left[\begin{array}{ll}
a_{k} & a_{k+1}
\end{array}\right]=1
$$

for each $k=1,2, \cdots, n-1$. Thus

$$
\operatorname{grid}_{a_{k}, a_{k+1}}^{\wedge}=\operatorname{grid}_{Q a_{k}, Q a_{k+1}}, k=0,1, \cdots, n-1
$$

(with $a_{n}:=a_{0}$ ) where

$$
Q=\left[\begin{array}{cc}
0 & -1 \\
1 & 0
\end{array}\right]
$$

is a quarter turn rotation. We will use this fact to generate quasiperiodic eigenfunctions of $\mathcal{F}$ on $\mathbb{R}^{2}$ with rotational symmetry.

We will now construct a family of quasiperiodic eigenfunctions of $\mathcal{F}$ that have rotational symmetry. Let $n=3,4, \cdots$, and $a_{k}, k=0,1,2, \cdots, n-1$ be given by (34), let $\alpha$ be given by (33), and let

$$
f_{n+}(x):=\sum_{k=0}^{n-1} \operatorname{grid}_{a_{k}, a_{k+1}}(x)+\operatorname{grid}_{a_{k}, a_{k+1}}^{\wedge}(x),
$$

and

$$
f_{n-}(x):=\sum_{k=0}^{n-1} \operatorname{grid}_{a_{k}, a_{k+1}}(x)-\operatorname{grid}_{a_{k}, a_{k+1}}^{\wedge}(x),
$$

(with $a_{n}:=a_{0}$ ). Figures 1 and 2 show representations of such eigenfunctions with $n=5$ and $n=7$ respectively. Filled circles correspond to negatively scaled Dirac $\delta$ 's, and unfilled circles correspond to positively scaled Dirac $\delta$ 's. The radius of each circle is proportional to the square root of the modulus of the scale factor for the corresponding $\delta$. By construction,

$$
f_{n+}^{\wedge}=f_{n+} \text { and } f_{n-}^{\wedge}=-f_{n-} .
$$

\section{Representation of Some Quasiperiodic Eigenfunctions}

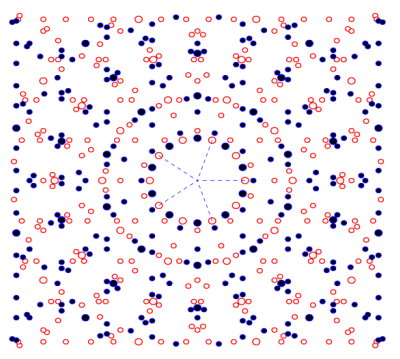

(a)

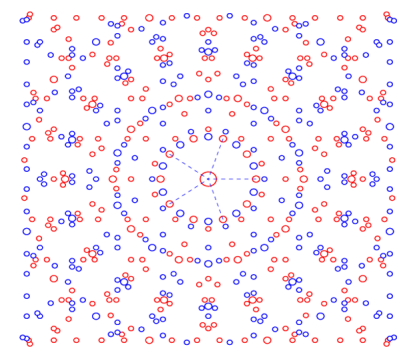

(b)
Figure 1. (a) $f_{5-}$; (b) $f_{5+}$; The quasiperiodic eigenfunctions $f_{5-}$ with 10-fold rotational symmetry, and $f_{5+}$ with 20-fold rotational symmetry for the Fourier transform operator $\mathcal{F}$ with respectively $\lambda=-1$, and $\lambda=1$. 


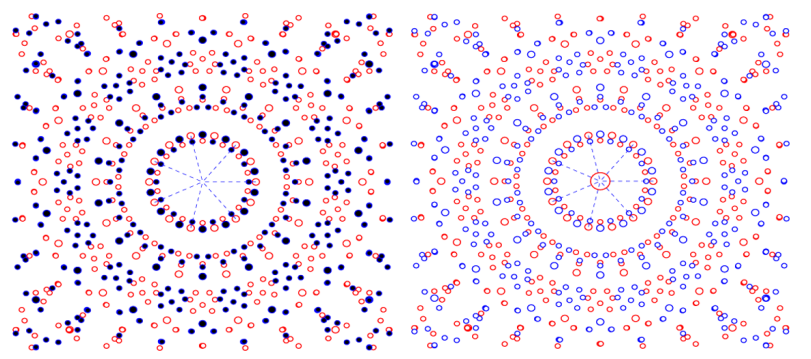

(a)

(b)

Figure 2. (a) $f_{7-}$; (b) $f_{7+}$; The quasiperiodic eigenfunctions $f_{7-}$ with 14-fold rotational symmetry, and $f_{7+}$ with 28-fold rotational symmetry for the Fourier transform operator $\mathcal{F}$ with respectively $\lambda=-1$, and $\lambda=1$.

\section{REFERENCES}

[1] R. N. Bracewell, "Fourier Analysis and Imaging," Kluwer Academic, New York, 2003. http://dx.doi.org/10.1007/978-1-4419-8963-5

[2] L. Schwartz, "Theorie des Distributions," Hermann, Paris, 1950.

[3] R. Strichartz, "A Guide to Distribution Theory and Fourier Transforms,” CRC Press, Inc., Boca Raton, 1994.
[4] B. Osgood, "The Fourier Transform and Its Applications," Lecture Notes, Stanford University, 2005.

[5] D. W. Kammler, "A First Course in Fourier Analysis," Prentice Hall, New Jersey, 2000.

[6] J. I. Richards and H. K. Youn, "Theory of Distributions: A Non-technical Introduction," Cambridge University Press, Cambridge, 1990. http://dx.doi.org/10.1017/CBO9780511623837

[7] M. J. Lighthill, "An Introduction to Fourier Analysis and Generalized Functions," Cambridge University Press, New York, 1958. http://dx.doi.org/10.1017/CBO9781139171427

[8] L. Auslander and R. Tolimieri, "Is Computing with the Finite Fourier Transform Pure or Applied Mathematics?" Bulletin of the American Mathematical Society, Vol. 1, 1979, pp. 847-897.

http://dx.doi.org/10.1090/S0273-0979-1979-14686-X

[9] J. H. McClellan and T. W. Parks, "Eigenvalue and Eigenvector Decomposition of the Discrete Fourier Transform," IEEE Transactions on Audio and Electroacoustics, Vol. 20, No. 1, 1972, pp. 66-74. http://dx.doi.org/10.1109/TAU.1972.1162342

[10] M. Senechal, "Quasicrystals and Geometry," Cambridge University Press, New York, 1995. 